\title{
Prognostic Value of Onodera's Nutritional Index for Intermediate and High Risk Gastrointestinal Stromal Tumors Treated With or Without Tyrosine Kinase Inhibitors
}

\section{feng wang}

Nanjing Drum Tower Hospital: Nanjing University Medical School Affiliated Nanjing Drum Tower Hospital

\section{Tingting Tao}

Nanjing Drum Tower Hospital: Nanjing University Medical School Affiliated Nanjing Drum Tower Hospital

\section{Heng Yu}

Nanjing Drum Tower Hospital: Nanjing University Medical School Affiliated Nanjing Drum Tower Hospital

\section{Yingying Xu}

Yangzhou University Medical college

\section{Zhi Yang}

Nanjing Drum Tower Hospital: Nanjing University Medical School Affiliated Nanjing Drum Tower Hospital

\section{Xuefeng Xia}

Nanjing Drum Tower Hospital: Nanjing University Medical School Affiliated Nanjing Drum Tower Hospital

\section{Meng Wang}

Nanjing Drum Tower Hospital: Nanjing University Medical School Affiliated Nanjing Drum Tower Hospital

\section{Liang Zong}

Changzhi People's Hospital https://orcid.org/0000-0003-4139-4571

\section{Wenxian Guan ( $\nabla$ medguanwenxian@163.com )}

Nanjing Drum Tower Hospital: Nanjing University Medical School Affiliated Nanjing Drum Tower Hospital

\section{Research}

Keywords: Gastrointestinal stromal tumor, Neutrophil-to-lymphocyte ratio, platelet-to-lymphocyte ratio, Onodera's prognostic nutritional index, propensity score matching, prognostic marker 
Posted Date: May 17th, 2021

DOI: https://doi.org/10.21203/rs.3.rs-127802/v3

License: (9) This work is licensed under a Creative Commons Attribution 4.0 International License. Read Full License

Version of Record: A version of this preprint was published at World Journal of Surgical Oncology on August 3rd, 2021. See the published version at https://doi.org/10.1186/s12957-021-02345-9. 


\section{Abstract}

Background: Immunoinflammatory and nutritional markers such as peripheral blood neutrophil-tolymphocyte ratio (NLR), platelet-to-lymphocyte ratio (PLR) and onodera's prognostic nutritional index (OPNI) have gained considerable attention and revealed preliminaryly as prognostic markers in gastrointestinal stromal tumor (GIST).

Methods: In this study, we firstly investigated the prognostic value of OPNI in GIST treated with or without TKIs based on the propensity score matching (PSM) method. All of the patients had received surgical resection for primary GIST, the data from 2010 to 2018 were initially and retrospectively identified from our gastrointestinal center. Recurrence-free survival (RFS) was calculated by the Kaplan-Meier method and compared by the log-rank test.

Results: These patients who were treated with TKIs and those who did not were divided into two groups, and we used propensity score matching method to make them have more unified baseline data. Multivariate Cox proportional hazard regression models were applied to identify associations with outcome variables. A total of 563 GISTs were initially chosen and 280 of them were included for analysis under an inclusion criteria. After PSM, there were 200 patients included. Multivariate analyses identified OPNI was an independent prognostic marker, and was associated with primary site, tumor size, mitotic index, tumor rupture, necrosis, and modified NIH risk classification. Low OPNI (<42.6; HR 0.409; P囚0.001) were associated with worse RFS.

Conclusions: Preoperative OPNI is a novel and useful prognostic marker for GISTs both treated with or without TKIs. Higher NLR and PLR have negative effects for RFS.

\section{Introduction}

Gastrointestinal stromal tumor (GIST) is the most common mesenchymal tissue neoplasms of digestive system. It often occurs in stomach and small intestine, accidentally found in abdominal and pelvic, omentum, colorectal, esophagus, pancreas, etc [1]. According to literatures, the incidence of GIST is about $0.001 \% \sim 0.0015 \%$ [2], which only accounts for a small part of gastrointestinal tumors. And the elderly suffer more. GIST is now considered to originate in the interstitial cell of Cajal, and the most common cause is mutations in receptor tyrosine kinases, especially among adults with proto-oncogene c-kit and platelet-derived growth factor receptor A (PDGFRA) [3]. Treatment methods for GIST are relatively limited because it is not sensitive to radiotherapy and chemotherapy, so surgical resection is the first choice, and is the only potentially curative therapy. Tyrosine kinase inhibitors (TKIs) are used as routine clinical drugs for GIST patients of medium to high risk due to their significant effects [4]. Despite the availability of TKIs such as imatinib mesylate (IM), which greatly promoted the disease-free survival (DFS), the relapse of GIST is common, even the tumors are R0 resected. So GIST is not easy to manage, let alone the prevalent side effects (fatigue, diarrhea, nausea, periorbital edema, muscle spasm, rash, etc) and resistance to IM. There are also approximately $15 \%$ of GIST patients are innately resistant or intolerant to first-line imatinib treatment[5-7].Therefore, accurate risk classification schemes are becoming increasingly significant for 
screening out patients who are most possibly to benefit from systematic IM therapy. Nowadays, four widely accepted factors which can reflect the prognosis of GIST patients are tumor location, size, mitotic index and tumor rupture as suggested by National Institutes of Health $(\mathrm{NIH})$ consensus criteria[8], Armed Forces Institute of Pathology (AFIP) criteria [9], and modified NIH consensus criteria [10]. As time goes by, more and more independent prognostic factors are proposed, such as antigen identified by monoclonal antibody Ki-67 index and surgery options[11, 12],

In addition, tumor-associated inflammatory cells, which consist tumor microenvironment, promote the proliferation, invasion and metastasis of tumor cells. Thus enhance the development and progression of tumor [13]. As many studies shown, GIST is also affected by immunoinflammatory factors such as peripheral blood neutrophil-to-lymphocyte ratio (NLR), as well as platelet-to-lymphocyte ratio (PLR)[14], which are readily measurable, reproducible and inexpensive systemic inflammatory marker. The that high level of NLR or PLR were reported to associate with poor prognosis of various solid tumors. However, investigations on the prognostic value of NLR and PLR for GISTs are lacking and the results remain controversial[15-17].Onodera's prognostic nutritional index (OPNI) was initially used to evalulate the immune-nutritional state of patients who are given gastrointestinal surgery [18]. Several studies have shown that the OPNI is a crucial prognostic factor in some specific human cancers such as gastric cancer [19], pancreatic cancer [20], colorectal cancer [21] and esophageal cancer [22]. Recently, an article about OPNI and GIST illustrated that OPNI plays a crucial role in prediction for GISTs that were not treated with medicine[23]. However, whether OPNI is a prognostic marker for GIST treated with TKIs has not been expounded, and the predictability difference between GIST treated with or without TKIs remains unknown. In this study, we investigate this firstly.

\section{Methods}

\section{Patients}

We retrospectively retrieved the 563 cases of GIST ranging from the lowest to high risk according to the modified NIH risk classification, in Nanjing Drum Tower Hospital from January 2010 to December 2018. Among them, 349 cases were not treated with TKIs, and the other 214 cases received TKIs therapy. In this study, we selected patients classified as the intermediate and high risk, and divided them into two groups: TKIs-using group and TKIs-unused group. We intended to investigate whether OPNI can be a prognostic marker to these two groups. The inclusion criteria was set as follows: (1)Classified as intermediate and high risk according to modified NIH risk classification; (2) primary localized GISTs with R0 resection; (3) no other synchronous primary tumors; (4) complete medical records; (5) patients whose follow-up was done. Eventually, 280 GISTs were enrolled in this investigation. Among them, 102 patients received no therapies of imatinib, while 178 patients were treated by imatinib after operation. This study was approved by the Ethics Committee of Nanjing Drum Tower Hospital. And written informed consent was acquired from all the patients in this program.

\section{Preoperative peripheral blood routine tests and OPNI evaluation}


All the results of preoperative peripheral blood routine and blood biochemistry were obtained within 5 days before surgery. The NLR value was calculated as neutrophil count $\left(10^{9} / \mathrm{L}\right)$ divided by the lymphocyte count $\left(10^{9} / \mathrm{L}\right)$. The value of platelet-to-lymphocyte ratio (PLR) was calculated same as NLR. The OPNI was calculated as serum albumin $(\mathrm{g} / \mathrm{L})+5 \times$ total lymphocyte count $\left(10^{9} / \mathrm{L}\right)$.

\section{Clinicopathological features}

All GISTs were initially diagnosed as gastrointestinal mesenchymal tumors by pathological ways based on a combination of histopathological evaluation and immunohistochemistry for CD117 or Discovered On GIST 1 (DOG1). They are further confirmed by CD34, desmin, SMA, S-100 expression. DNA mutation analysis of PDGFRA gene exons 12 and 18 or c-kit gene exons 9,11,13 and 17 were also made partly to determine the application of TKIs. In this study, clinical data and histopathological parameters are all collected from medical records. Clinical data includes age, gender, initial complaint, primary tumor site, tumor size, surgery options, tumor rupture (preoperative or intraoperative), whether the TKIs were used and hospitalization time. Tumor size was accurately measured by pathologists after surgery. Histopathological factors include predominant cell type (spindle, epithelioid, or mixed), mitotic index (per 50 randomly selected high power fields [HPFs]), tumor necrosis and Ki-67 index. Risk stratification of each case was determined by modified NIH consensus criteria covering tumor size, mitotic index, tumor site, and rupture.

\section{Follow-up}

The patients after surgery were followed up through routine peripheral blood tests, abdominal ultrasonography, endoscopy and computed tomography (CT) every 6 months in the first 5 years, and then annually after 5 years to evaluate tumor recurrence or distant metastasis. Follow-up information was obtained by outpatient or hospitalized records, or direct contact with patients or their family. All of the clinical data and follow-up job was accomplished by professional staff in our department. Relapse-free survival (RFS) is more suitable to evaluate patients' survival than overall survival (OS). RFS was calculated from the date of surgery to the date of GIST relapse, metastasize or to the last follow-up date. Median follow-up time was estimated by Kaplan-Meier method.

\section{Statistical analysis}

All statistical analyses were calculated by using IBM SPSS Statistics, version 22.0 (IBM, New York, USA) and $\mathrm{R}$ 3.6.3. The ranked and unordered categorical variables were respectively assessed by MannWhitney $\mathrm{U}$ and Chi-square test. The correlation of continuous variables was calculated by Pearson correlation coefficient, while discrete variables by Spearman's correlation coefficient. Cox's regression model was used to perform multivariate survival analyses. The log-rank test and Kaplan-Meier method were utilized to calculate univariate survival. The PLR, NLR, OPNI cut-off value was determined by R 3.6.3 which was performed based on the recurrence state at 9-year follow-up. A P-value $<0.05$ was indicated to be statistical significant, and confidence intervals $(\mathrm{Cl})$ were calculated at the $95 \%$ level. 
In this study, we applied 1:1 propensity score matching to adjust patients for gender, age, primary tumor site, tumor size, mitotic index and risk stratification in order to reduce the effect of potential confounding factors and selection bias, such as patients' baseline clinicopathologic factors or unequal patients distribution between the TKIs-used and TKIs-unused groups. We applied the nearest neighbor matching method and a 0.05 -width caliper of the standard deviation of the logit was set to match the two groups.

\section{Results}

The median age of 280 patients was 60 years old (range 26 to 83 years old), with 114 patients $(40.7 \%)$ aged $>60$ years. Among them, there were 143 men and 137 women. Primary manifestations of GISTs were as follows: abdominal discomfort or pain $(n=65)$, Gl bleeding $(n=56)$, obstruction $(n=17)$, tumor perforation or rupture $(n=24)$, medical examination reported $(n=104)$, and other symptoms $(n=14)$. The primary tumor sites were mainly stomach $(n=182)$, secondly small intestine $(n=84)$, and colorectum or intraperitoneally with unknown origin in the next place $(n=14)$. The tumor size varied from 1.0 to $30.0 \mathrm{~cm}$ (median, $7.5 \mathrm{~cm})$. Histologically, the spindle cell type was most common $(n=162)$, followed by epithelioid cell type $(n=12)$ and mixed type $(n=6)$. The mitotic index, necrosis, and more detailed clinicopathological variables of our patients before and after PSM are summarized in Table 1. 
Table 1

Clinicopathological features of 280 patients with primary GIST that classified as medium and high risk.

\begin{tabular}{|c|c|c|c|c|c|c|}
\hline \multirow[t]{2}{*}{ Characteristics } & \multicolumn{3}{|c|}{ Before matching $(n=280)$} & \multicolumn{3}{|c|}{ After matching $(n=200)$} \\
\hline & $\begin{array}{l}\text { TKls-used } \\
\text { group }(n= \\
178)\end{array}$ & $\begin{array}{l}\text { TKIs-unused } \\
\text { group }(n= \\
102)\end{array}$ & $\begin{array}{l}P \\
\text { value }\end{array}$ & $\begin{array}{l}\text { TKls-used } \\
\text { group }(n= \\
100)\end{array}$ & $\begin{array}{l}\text { TKIs-unused } \\
\text { group }(n= \\
100)\end{array}$ & $\begin{array}{l}\mathrm{P} \\
\text { value }\end{array}$ \\
\hline Gender & & & 0.446 & & & 0.257 \\
\hline Male(\%) & $100(56.2)$ & $43(42.2)$ & & $52(52.00)$ & $43(43.00)$ & \\
\hline Female(\%) & $78(43.8)$ & $59(57.8)$ & & $48(48.00)$ & $57(57.00)$ & \\
\hline Age & & & 0.064 & & & \\
\hline$\leq 60$ years & $116(65.2)$ & $50(49.0)$ & & $52(52.00)$ & $50(50.00)$ & \\
\hline$>60$ years & $62(34.8)$ & $52(51.0)$ & & $48(48.00)$ & $50(50.00)$ & \\
\hline $\begin{array}{l}\text { Clinical } \\
\text { manifestation }\end{array}$ & & & 0.469 & & & 0.362 \\
\hline $\begin{array}{l}\text { Abdominal } \\
\text { discomfort or } \\
\text { pain(\%) }\end{array}$ & $41(23.03)$ & $24(23.53)$ & & $36(36.00)$ & $39(39.0)$ & \\
\hline $\begin{array}{l}\text { Gastrointestinal } \\
\text { bleeding(\%) }\end{array}$ & $36(20.22)$ & 20(19.61) & & 17(17.00) & 19(19.00) & \\
\hline Obstruction & $11(6.18)$ & $6(5.88)$ & & $2(2.00)$ & $2(2.00)$ & \\
\hline $\begin{array}{l}\text { Perforation or } \\
\text { rupture(\%) }\end{array}$ & 14(7.87) & $10(9.80)$ & & $8(8.00)$ & $9(9.00)$ & \\
\hline $\begin{array}{l}\text { Medical } \\
\text { examination } \\
\text { reported(\%) }\end{array}$ & 68(38.20) & $36(35.29)$ & & $32(32.00)$ & $27(27.00)$ & \\
\hline Others(\%) & $8(4.49)$ & $6(5.88)$ & & $5(5.00)$ & $4(4.00)$ & \\
\hline \multicolumn{7}{|l|}{$\begin{array}{l}\text { Preoperative } \\
\text { laboratory } \\
\text { variables }\end{array}$} \\
\hline $\begin{array}{l}\text { Hemoglobin }(\mathrm{g} / \mathrm{L}, \\
x \pm \mathrm{s})\end{array}$ & $113.3 \pm 22.3$ & $108.5 \pm 26.4$ & 0.206 & $116.3 \pm 25.6$ & $108.3 \pm 26.5$ & \\
\hline $\begin{array}{l}\text { White blood cell } \\
\left(10^{9} / L, ' x \pm s\right)\end{array}$ & $6.4 \pm 2.5$ & $6.7 \pm 4.2$ & 0.662 & $6.4 \pm 2.5$ & $6.7 \pm 4.2$ & \\
\hline $\begin{array}{l}\text { Neutrophil count } \\
\left(10^{9} / L,{ }^{\prime} x \pm s\right)\end{array}$ & $4.4 \pm 3.2$ & $4.5 \pm 4.0$ & 0.809 & $4.2 \pm 2.3$ & $4.6 \pm 4.0$ & \\
\hline $\begin{array}{l}\text { Lymphocyte count } \\
\left(10^{9} / L, ' x \pm s\right)\end{array}$ & $1.5 \pm 0.6$ & $1.6 \pm 1.4$ & 0.397 & $1.6 \pm 0.6$ & $1.6 \pm 1.4$ & \\
\hline
\end{tabular}




\begin{tabular}{|c|c|c|c|c|c|c|}
\hline \multirow[t]{2}{*}{ Characteristics } & \multicolumn{3}{|c|}{ Before matching $(n=280)$} & \multicolumn{3}{|c|}{ After matching $(n=200)$} \\
\hline & $\begin{array}{l}\text { TKIs-used } \\
\text { group }(n= \\
178)\end{array}$ & $\begin{array}{l}\text { TKIs-unused } \\
\text { group }(n= \\
102)\end{array}$ & $\begin{array}{l}P \\
\text { value }\end{array}$ & $\begin{array}{l}\text { TKls-used } \\
\text { group }(n= \\
100)\end{array}$ & $\begin{array}{l}\text { TKls-unused } \\
\text { group }(n= \\
100)\end{array}$ & $\begin{array}{l}\mathrm{P} \\
\text { value }\end{array}$ \\
\hline $\begin{array}{l}\text { Platelet count } \\
\left(10^{9} / \mathrm{L}, ' x \pm \mathrm{s}\right)\end{array}$ & $230.4 \pm 87.7$ & $237.8 \pm 103.3$ & 0.616 & $226.6 \pm 78.4$ & $237.4 \pm 104.4$ & \\
\hline $\begin{array}{l}\text { Albumin (g/L, ' } x \pm \\
\mathrm{s})\end{array}$ & $38.9 \pm 4.3$ & $38.9 \pm 4.8$ & 0.992 & $39.0 \pm 4.0$ & $38.9 \pm 4.9$ & \\
\hline $\operatorname{NLR}(x \pm s)$ & $4.0 \pm 5.5$ & $3.9 \pm 5.1$ & 0.939 & $3.5 \pm 3.8$ & $3.9 \pm 5.1$ & \\
\hline $\operatorname{PLR}(x \pm s)$ & $182.7 \pm 136.1$ & $194.2 \pm 143.3$ & 0.339 & $170.3 \pm 121.6$ & $193.9 \pm 143.9$ & \\
\hline OPNI $(x \pm s)$ & $46.4 \pm 5.7$ & $46.4 \pm 8.6$ & 0.015 & $47.2 \pm 5.7$ & $46.4 \pm 8.6$ & \\
\hline Primary tumor site & & & $<0.001$ & & & 0.137 \\
\hline Stomach(\%) & 114(64.04) & $68(66.67)$ & & $75(75.00)$ & $66(66.00)$ & \\
\hline Small intestine(\%) & $54(30.34)$ & $30(29.41)$ & & $20(20.00)$ & $30(30.00)$ & \\
\hline Colorectum(\%) & $3(1.69)$ & $2(1.96)$ & & $2(2.00)$ & $2(2.00)$ & \\
\hline $\begin{array}{l}\text { Intraperitoneally } \\
\text { with unknown } \\
\text { origin(\%) }\end{array}$ & $7(3.93)$ & $2(1.96)$ & & $3(3.00)$ & $2(2.00)$ & \\
\hline $\begin{array}{l}\text { Tumor size }\left(\mathrm{cm},{ }^{\prime} x\right. \\
\pm \mathrm{s})\end{array}$ & $7.75 \pm 3.64$ & $7.17 \pm 4.45$ & 0.051 & $6.67 \pm 2.75$ & $7.16 \pm 4.49$ & 0.469 \\
\hline$\leq 5.0$ & $32(17.98)$ & $27(26.47)$ & & $23(23.00)$ & $26(26.00)$ & \\
\hline $5.1-10.0$ & 112(62.92) & $62(60.78)$ & & $72(72.00)$ & $61(61.00)$ & \\
\hline$>10.0$ & $34(19.10)$ & $13(12.75)$ & & $5(5.00)$ & $13(13.00)$ & \\
\hline $\begin{array}{l}\text { Predominant cell } \\
\text { type }\end{array}$ & & & 0.685 & & & 0.795 \\
\hline Spindle & $170(19.50)$ & $92(90.30)$ & & $95(95.00)$ & $90(90.00)$ & \\
\hline Epithelioid & $5(2.81)$ & $7(6.86)$ & & $4(4.00)$ & $7(7.00)$ & \\
\hline Mixed & $3(1.69)$ & $3(2.94)$ & & $1(1.00)$ & $3(3.00)$ & \\
\hline $\begin{array}{l}\text { Mitotic index (per } \\
50 \text { HPFs) }\end{array}$ & & & 0.875 & & & 0.250 \\
\hline$\leq 5(\%)$ & $85(47.75)$ & $47(46.08)$ & & $53(53.00)$ & $45(45.00)$ & \\
\hline $6-10(\%)$ & $35(19.66)$ & $26(25.49)$ & & $21(21.00)$ & $26(26.00)$ & \\
\hline$>10(\%)$ & $58(32.58)$ & $29(28.43)$ & & $26(26.00)$ & $29(29.00)$ & \\
\hline
\end{tabular}




\begin{tabular}{|c|c|c|c|c|c|c|}
\hline \multirow[t]{2}{*}{ Characteristics } & \multicolumn{3}{|c|}{ Before matching $(n=280)$} & \multicolumn{3}{|c|}{ After matching $(n=200)$} \\
\hline & $\begin{array}{l}\text { TKls-used } \\
\text { group }(n= \\
\text { 178) }\end{array}$ & $\begin{array}{l}\text { TKls-unused } \\
\text { group }(n= \\
\text { 102) }\end{array}$ & $\begin{array}{l}\mathrm{P} \\
\text { value }\end{array}$ & $\begin{array}{l}\text { TKls-used } \\
\text { group }(n= \\
100)\end{array}$ & $\begin{array}{l}\text { TKls-unused } \\
\text { group }(n= \\
100)\end{array}$ & $\begin{array}{l}P \\
\text { value }\end{array}$ \\
\hline Necrosis & & & 0.014 & & & 0.002 \\
\hline Yes (\%) & $62(34.83)$ & $32(31.37)$ & & 33(33.00) & $37(37.00)$ & \\
\hline No (\%) & $116(65.17)$ & $70(68.63)$ & & $67(67.00)$ & $63(63.00)$ & \\
\hline Tumor rupture & & & $<0.001$ & & & $\begin{array}{l}<.001 \\
0.001\end{array}$ \\
\hline Yes(\%) & $23(12.92)$ & 14(13.73) & & $6(6.00)$ & $7(7.00)$ & \\
\hline No (\%) & 155(87.08) & $88(86.27)$ & & $94(94.00)$ & $93(93.00)$ & \\
\hline Risk classification & & & 0.096 & & & 0.024 \\
\hline $\begin{array}{l}\text { Intermediate } \\
\text { risk(\%) }\end{array}$ & $59(33.15)$ & $44(43.14)$ & & $58(58.00)$ & $42(42.00)$ & \\
\hline High risk(\%) & $119(66.85)$ & $58(56.86)$ & & $42(42.00)$ & $58(58.00)$ & \\
\hline CD117 & & & 0.279 & & & 0.031 \\
\hline$(-)(\%)$ & $3(1.69)$ & $5(4.90)$ & & $2(2.00)$ & $5(5.00)$ & \\
\hline$(+)(\%)$ & $37(20.79)$ & $27(26.47)$ & & $23(23.00)$ & $25(25.00)$ & \\
\hline$(++)(\%)$ & $35(19.66)$ & $10(9.80)$ & & $17(17.00)$ & $11(11.00)$ & \\
\hline$(+++)(\%)$ & 103(57.87) & $60(58.82)$ & & $58(58.00)$ & $59(59.00)$ & \\
\hline CD34 & & & 0.257 & & & 0.531 \\
\hline$(-)(\%)$ & $20(11.24)$ & 12(11.76) & & $10(10.00)$ & $12(12.00)$ & \\
\hline$(+)(\%)$ & $33(18.54)$ & $36(35.29)$ & & $15(15.00)$ & $33(33.00)$ & \\
\hline$(++)(\%)$ & $22(12.36)$ & $8(7.84)$ & & $12(12.00)$ & $8(8.00)$ & \\
\hline$(+++)(\%)$ & 103(57.87) & $46(45.10)$ & & $63(63.00)$ & $46(46.00)$ & \\
\hline $\begin{array}{l}\text { Ki-67 index (\%, ` } x \\
\pm s)\end{array}$ & $7.87 \pm 7.66$ & $7.31 \pm 7.59$ & 0.019 & $7.01 \pm 6.62$ & $7.35 \pm 7.69$ & 0.766 \\
\hline$\leq 5(\%)$ & $110(61.80)$ & $64(62.75)$ & & $67(67.00)$ & $63(63.00)$ & \\
\hline $6-10(\%)$ & $35(19.66)$ & $25(24.51)$ & & $16(16.00)$ & $24(24.00)$ & \\
\hline$>10(\%)$ & $33(18.54)$ & $13(12.75)$ & & $17(17.00)$ & $13(13.00)$ & \\
\hline $\begin{array}{l}\text { Follow-up time } \\
\text { (months, } x \pm s \text { ) }\end{array}$ & $44.47 \pm 25.07$ & $55.01 \pm 29.39$ & 0.003 & $48.57 \pm 24.61$ & $53.98 \pm 28.75$ & $\dot{0}_{0.001}$ \\
\hline
\end{tabular}




\begin{tabular}{|c|c|c|c|c|c|c|}
\hline \multirow[t]{2}{*}{ Characteristics } & \multicolumn{3}{|c|}{ Before matching $(n=280)$} & \multicolumn{3}{|c|}{ After matching $(n=200)$} \\
\hline & $\begin{array}{l}\text { TKls-used } \\
\text { group }(n= \\
178)\end{array}$ & $\begin{array}{l}\text { TKls-unused } \\
\text { group }(n= \\
\text { 102) }\end{array}$ & $\begin{array}{l}P \\
\text { value }\end{array}$ & $\begin{array}{l}\text { TKls-used } \\
\text { group }(n= \\
100)\end{array}$ & $\begin{array}{l}\text { TKls-unused } \\
\text { group }(n= \\
100)\end{array}$ & $\begin{array}{l}\mathrm{P} \\
\text { value }\end{array}$ \\
\hline Follow-up status & & & $<_{0.001}$ & & & $\begin{array}{l}< \\
0.001\end{array}$ \\
\hline $\begin{array}{l}\text { Relapse-free } \\
\text { survival(\%) }\end{array}$ & 146(82.02) & $72(70.59)$ & & $85(85.00)$ & $70(70.00)$ & \\
\hline Relapse (\%) & $32(17.98)$ & $30(29.41)$ & & $15(15.00)$ & $30(30.00)$ & \\
\hline
\end{tabular}

According to the recent study, OPNI is a prognostic marker to GIST[23]. We used the continuous variable NLR, PLR and OPNI of 200 patients after PSM, and their RFS and outcome as the state variable. The Hosmer-Lemeshow test and the value of c-statistic ( 0.71$)$ showed fairly excellent calibration $(p=0.08)$ and discrimination, respectively, between the 2 groups. The ASD values after matching ranged from 0 to 8\%. The cut-off point of OPNI is $42.6(P<0.001)$, NLR is 5.1 $(P<0.001)$ and PLR is 98.6 $(P=0.008)$. Exp (coef), univariate P-value and Hazard ratio of NLR, PLR and OPNI were summarized in Table 2, Fig. 1.

Table 2

analysis for NLR, PLR, OPNI.

\begin{tabular}{|llll|}
\hline & NLR & PLR & OPNI \\
\hline Exp(coef) & 1.038 & 1.002 & 0.933 \\
\hline Univariate P-value & 0.129 & 0.084 & 0.002 \\
\hline Best cut-off point & 5.1 & 98.6 & 42.6 \\
\hline Hazard ratio & 3.000 & 1.795 & 0.315 \\
\hline Log rank P & $<0.001$ & 0.008 & $<0.001$ \\
\hline
\end{tabular}

As we saw in Spearman Correlation analysis, higher OPNI was associated with primary tumor site of stomach $(P<0.01)$, smaller tumor size $(P<0.017)$, lower mitotic index $(P<0.001)$, lower modified $N I H$ risk classification $(P<0.001)$, less gastrointestinal bleeding rate $(P<0.01)$ and tumor rupture $(P<0.01)$ and much lower tumor relapse rate $(P<0.01)$. A strong correlation was observed between NLR and tumor site $(P=0.01)$, $G$ bleeding $(P<0.01)$, tumor rupture $(P=0.03)$ and relapse $(P=0.02)$. And $P L R$ was also connected with tumor site $(P<0.01)$, Gl bleeding $(P=0.04)$ and relapse $(P=0.03)$. (Table 3, Fig. 2 and Table 4) 
Table 3

Correlation analysis of tumor size and mitotic index with NLR,

PLR, OPNI and Ki-67 index.

\begin{tabular}{|lllll|}
\hline & Tumor size & \multicolumn{3}{c|}{ Mitotic index } \\
\hline & Spearman $r$ & P-value & Spearman $r$ & P-value \\
\hline NLR & 0.06 & $<0.01$ & 0.11 & 0.07 \\
\hline PLR & 0.28 & $<0.01$ & 0.15 & 0.01 \\
\hline OPNI & -0.27 & $<0.01$ & -0.14 & 0.02 \\
\hline Ki-67 index & 0.35 & 0.02 & 0.44 & $<0.01$ \\
\hline
\end{tabular}

Table 4

Correlation analysis of OPNI, NLR and PLR with tumor site, gastrointestinal bleeding (GI bleeding), tumor rupture, relapse, CD34 and CD117.

\begin{tabular}{|lllllll|}
\hline Factors & OPNI & \multicolumn{3}{c|}{ NLR } & \multicolumn{3}{l|}{ PLR } \\
\cline { 2 - 7 } & Spearman r & P-value & Spearman r & P-value & Spearman r & P-value \\
\hline Site & -0.26 & $<0.01$ & 0.18 & 0.01 & 0.19 & $<0.01$ \\
\hline Gl bleeding & -0.26 & $<0.01$ & 0.20 & $<0.01$ & 0.15 & 0.04 \\
\hline Tumor rupture & -0.13 & 0.06 & 0.15 & 0.03 & 0.12 & 0.09 \\
\hline Relapse & -0.23 & $<0.01$ & 0.16 & 0.02 & 0.15 & 0.03 \\
CD34 & -0.07 & 0.26 & 0.02 & 0.74 & 0.07 & 0.27 \\
\hline CD117 & -0.06 & 0.36 & -0.01 & 0.81 & 0.05 & 0.38 \\
\hline
\end{tabular}

Patients were followed for a median of 48 months (range: 8months-103months). This was calculated by Kaplan-Meier method, we concluded that our estimated median follow-up time was 47.98 months $(\mathrm{P}<$ 0.001). 62 patients experienced tumor relapse during the follow-up period. There were 30 of them had not been treated by IM, and 32 of them had received IM therapy with a duration ranged from 1 months to 5 years. Until December 2018, 13 patients without medication had died, with a mortality rate of $12.7 \%$ $(12 / 102)$, while the mortality rate in the drug treatment group was $3.3 \%(6 / 178)$. Metastasis to the lymph nodes was not spotted.

Our univariate survival analysis showed that tumor size (Log-rank $P=0.002)$, mitotic index (Log-rank $P<$ $0.001)$, modified NIH risk stratification (Log-rank $P<0.001)$, NLR (Log-rank $P=0.002), P L R($ Log-rank $P=$ 0.004), , primary tumor site(Log-rank $P=0.007)$, age (Log-rank $P=0.023)$ and OPNI (Log-rank $P=0.045)$ were all significant prognostic parameters for RFS. Results of univariate survival analysis are in Table 5. Some sorted factors were analyzed in the Cox proportional hazards model in enter strategies. The results of the Cox regression analysis are listed in Table 5. High mitotic index $(P=0.002)$, age more than $60(P=$ $0.008)$, larger tumor size $(P=0.003)$, high NLR $(P=0.031)$, and low OPNI $(P=0.009)$ were statistically significant independent negative prognostic indicators for RFS. 
Table 5

Univariate and multivariate analysis of the prognostic factors for recurrence-free survival of patients after PS matching.

\begin{tabular}{|c|c|c|c|c|}
\hline \multirow[t]{2}{*}{ Characteristics } & \multicolumn{2}{|c|}{ Univariate analysis } & \multicolumn{2}{|l|}{ Multivariate analysis } \\
\hline & $\mathrm{HR}(95 \% \mathrm{Cl})$ & $\begin{array}{l}P \\
\text { value }\end{array}$ & $\mathrm{HR}(95 \% \mathrm{Cl})$ & $\begin{array}{l}P \\
\text { value }\end{array}$ \\
\hline Age/year & & 0.023 & & 0.008 \\
\hline$\leq 60$ & 1 & & 1 & \\
\hline$₫ 60$ & $\begin{array}{l}1.910(1.095- \\
3.333)\end{array}$ & & $2.175(1.226-3.859)$ & \\
\hline Gender & & 0.064 & & \\
\hline Male & 1 & & & \\
\hline Female & $\begin{array}{l}0.533(0.331- \\
1.120)\end{array}$ & & & \\
\hline GI bleeding & & 0.337 & & \\
\hline Yes & 1 & & & \\
\hline No & $\begin{array}{l}1.307(0.756- \\
2.261)\end{array}$ & & & \\
\hline Primary site & & 0.007 & & 0.864 \\
\hline Gastric & 1 & & 1 & \\
\hline Non-gastric & $\begin{array}{l}2.116(1.228- \\
3.645)\end{array}$ & & $1.066(0.560-2.029)$ & \\
\hline Tumor size & & 0.002 & & 0.003 \\
\hline$\leq 5.0 \mathrm{~cm}$ & 1 & & 1 & \\
\hline$>5.0 \mathrm{~cm}$ & $\begin{array}{l}1.962(1.254- \\
2.848)\end{array}$ & & $1.758(1.067-3.759)$ & \\
\hline Predominant cell type & & 0.419 & & \\
\hline Spindle & 1 & & & \\
\hline Epithelioid & $\begin{array}{l}0.765(0.263- \\
3.376)\end{array}$ & & & \\
\hline Mixed & $\begin{array}{l}0.735(0.363- \\
3.289)\end{array}$ & & & \\
\hline Mitotic index & & $\hat{0.001}$ & & 0.002 \\
\hline$\leq 5$ per $50 \mathrm{HPFs}$ & 1 & & 1 & \\
\hline
\end{tabular}




\begin{tabular}{|c|c|c|c|c|}
\hline \multirow[t]{2}{*}{ Characteristics } & \multicolumn{2}{|c|}{ Univariate analysis } & \multicolumn{2}{|l|}{ Multivariate analysis } \\
\hline & $\mathrm{HR}(95 \% \mathrm{Cl})$ & $\begin{array}{l}P \\
\text { value }\end{array}$ & $\mathrm{HR}(95 \% \mathrm{Cl})$ & $\begin{array}{l}P \\
\text { value }\end{array}$ \\
\hline $6-10$ per $50 \mathrm{HPFs}$ & $\begin{array}{l}1.524(1.186- \\
2.654)\end{array}$ & & $1.387(1.084-2.368)$ & \\
\hline$>10$ per $50 \mathrm{HPFs}$ & $\begin{array}{l}2.098(1.522- \\
2.890)\end{array}$ & & $1.811(1.247-2.629)$ & \\
\hline Tumor rupture & & 0.097 & & \\
\hline No & 1 & & & \\
\hline Yes & $\begin{array}{l}1.801(0.711- \\
4.563)\end{array}$ & & & \\
\hline NIH risk classification & & $<.001$ & & 0.024 \\
\hline Intermediate risk & 1 & & 1 & \\
\hline High risk & $\begin{array}{l}4.943(2.479- \\
9.854)\end{array}$ & & $2.640(1.140-6.115)$ & \\
\hline Ki-67 index & & 0.181 & & \\
\hline$\leq 5$ & 1 & & & \\
\hline $6-10$ & $\begin{array}{l}1.128(0.581- \\
3.835)\end{array}$ & & & \\
\hline$>10$ & $\begin{array}{l}1.045(0.854- \\
4.185)\end{array}$ & & & \\
\hline NLR & & 0.002 & & 0.031 \\
\hline$<5.1$ & 1 & & 1 & \\
\hline$\geq 5.1$ & $\begin{array}{l}1.552(1.180- \\
2.041)\end{array}$ & & $1.878(1.060-3.327)$ & \\
\hline PLR & & 0.004 & & 0.407 \\
\hline$<98.6$ & 1 & & 1 & \\
\hline$\geq 98.6$ & $\begin{array}{l}1.453(1.126- \\
1.875)\end{array}$ & & $1.262(0.727-2.191)$ & \\
\hline OPNI & & 0.045 & & 0.009 \\
\hline$<42.6$ & 1 & & 1 & \\
\hline$\geq 42.6$ & $\begin{array}{l}0.409(0.170- \\
0.980)\end{array}$ & & $0.592(0.400-0.875)$ & \\
\hline
\end{tabular}


We divided patients with or without medical treatment into several groups according to relatively low or high OPNI, NLR and PLR. And we observed that patients who used TKIs had a better relapse free survival. In addition, patients with higher OPNI showed preferable RFS than those with lower OPNI $(P<0.0001)$. Furthermore, higher NLR $(P<0.0001)$ and PLR $(P=0.0003)$ were factors leading to poor prognosis (Fig. 3).

\section{Discussion}

In this study, according to recent investigations of Sun JY's team that showed OPNI was an independent predictive factor of RFS in GIST patients with no TKIs treatment [23]. We initially base our survival cut-off analysis on the 200 GIST patients with median or high risk after PSM and get the best cut-off points of NLR, PLR and OPNI. Then we examined the univariate and multivariate survival analysis of our patients after PSM study. It was our aim to investigate the prognostic value of OPNI in intermediate and high risk gastrointestinal stromal tumors treated with or without TKIs. Eventually, analysis proved that OPNI was an independent prognostic marker for both two groups.

A more precise risk classification criterion that can be applied to determine the postoperative prognosis of patients with GIST is eagerly required. Of which the items should be simply and economically detected and calculated by clinicopathological data. Nowadays, the most widely used criterion to estimate the risk of relapse after surgery in GIST are the AFIP criteria, and modified NIH consensus criteria. Studies have proved that their prognostic accuracy is similar, by and large [24]. Moreover, Memorial Sloan-Kettering Cancer Center sarcoma team developed a nomogram that could estimate the probability of RFS at 2 and 5-year after surgery for primary GIST and was more precise than NIH criteria to a certain extent [25]. Joensuu $\mathrm{H}$ further demonstrated the KIT and PDGFRA mutations may have widely varying risks for recurrence, and those with KIT exon 11 duplication mutation or deletion of one codon have favorable RFS with surgery alone [26].

OPNI is a nutrition index which is firstly raised by Onodera and his colleagues. The previous studies showed that patients with high OPNI shared a significantly better prognosis than those who had a lower value of OPNI [22]. And similar results regarding Crohn's disease and stage III colorectal cancer have been also reported $[27,28]$. In our study, the border value of the OPNI was determined to be 42.6 for TKIs-used group and TKIs-unused group according to survival cut-off analysis by R 3.6.3. A detailed analysis demonstrated that higher OPNI was associated with primary tumor site of stomach, smaller tumor size, lower mitotic index, lower modified NIH risk classification, better gastrointestinal bleeding rate and tumor rupture and much lower tumor relapse rate. In univariate and multivariate survival analysis, OPNI was independent prognostic indicators too. Lower OPNI may result from low hypoproteinemia and/or lymphopenia, which can be explained by several potential phenomena: (1) nutritional supplementation of branched-chain amino acids can improve hypoproteinemia and reduce tumor recurrence in patient ; 2 ) lymphocytes play an important role in the host immune response, eliminating tumor formation and progression. Postoperative follow-up examination for OPNI is also recommended, yet we were lack of this data. 
In our univariate and multivariate analysis of the prognostic factors for recurrence-free survival of patients after PS matching, we found that high mitotic index, age more than 60-year-old, larger tumor size, high NLR, and low OPNI were statistically significant independent negative prognostic indicators for RFS. Moreover, when we had split patients into groups according to the best cut-off value of NLR, PLR and OPNI, we found that relatively lower NLR, lower PLR and higher OPNI were significantly linked to better RFS.

There does exist limitations of this study. Firstly, it is a single-center retrospective study, therefore, a multicenter study is eagerly required to enlarge the sample to minimize the deficiency during the analysis. Secondly, the best cut-off value in this study is determined by survival cut-off analysis. However, it is still unclear what cut-off value is the best optimal cut-off value for clinical diagnosis of GIST due to the limited amount of patients. Thirdly, mutations were not well considered because there were just part of patients had gene test. In general, exploring the exact best cut-off value and studying its intrinsic molecular mechanism will be the future research direction.

In conclusion, we found connection among immuno-inflammatory factors (NLR and PLR), nutritional factors (OPNI), clinico-pathological characteristics and the RFS of intermediate and high risk GIST treated with or without TKIs. OPNI is an independent indicator for RFS in GIST treated with or without TKIs. Furthermore, OPNI also might be a ponderable factor for predicting tumor biological behavior from peripheral blood.

\section{Abbreviations}

$\mathrm{NLR}=$ neutrophil-to-lymphocyte ratio

PLR = platelet-to-lymphocyte ratio

$\mathrm{OPNI}=$ onodera's prognostic nutritional index

GIST = gastrointestinal stromal tumors

PSM = propensity score matching

RFS $=$ recurrence-free survival

TKIs $=$ Tyrosine kinase inhibitors

$\mathrm{IM}=$ imatinib mesylate

NCCN $=$ National Comprehensive Cancer Network

$\mathrm{NIH}=$ National Institutes of Health

AFIP = Armed Forces Institute of Pathology 
DFS $=$ disease-free survival

OS = overall survival

PDGFRA = platelet-derived growth factor receptor $\mathrm{A}$

HPFs $=$ high power fields

$\mathrm{CT}=$ computed tomography

$\mathrm{ROC}=$ receiver operating characteristic

$\mathrm{Cl}=$ confidence intervals

\section{Declarations}

\section{Ethics approval and consent to participate}

This study has been approved by the Ethics Committees of Nanjing Drum Tower Hospital.

\section{Consent for publication}

No

\section{Availability of data and materials}

Access to the data and the calculation method can be obtained from the authors by email (fengwang36@163.com).

\section{Competing of Interests}

The authors declare that they have no competing of interests.

\section{Funding}

This work was supported by Jiangsu Provincial Key Research and Development Program (social development project) , China (No. BE2016603)

\section{Authors' contributions}

FW, TT and LZ contributed to the study design, drafted the manuscript. HY and ZY worked on study design and data analysis. $X X$ and $Y X$ were involved in data collection and extraction. MW, LZ and WG revised the manuscript. All authors have read and approved the final manuscript.

\section{Acknowledgments}

The authors gratefully acknowledge all of the investigators for their contributions to the trial. 


\section{References}

1. Keung EZ, Raut CP. :Management of Gastrointestinal Stromal Tumors. Surg Clin North Am. 2017;97(2):437-52.

2. Nishida T, Goto O, Raut CP, Yahagi N. Diagnostic and treatment strategy for small gastrointestinal stromal tumors. Cancer. 2016;122(20):3110-8.

3. Heinrich MC, Corless CL, Duensing A, McGreevey L, Chen CJ, Joseph N, Singer S, Griffith DJ, Haley A, Town A, et al: PDGFRA activating mutations in gastrointestinal stromal tumors. Science 299(5607):708-710,2003.

4. Seifert AM, Zeng S, Zhang JQ, Kim TS, Cohen NA, Beckman MJ, Medina BD, Maltbaek JH, Loo JK, Crawley MH, et al:PD-1/PD-L1 Blockade Enhances T-cell Activity and Antitumor Efficacy of Imatinib in Gastrointestinal Stromal Tumors. Clinical Cancer Research 23(2):454-465,2016.

5. Sodergren SC, White A, Efficace F, Sprangers M, Fitzsimmons D, Bottomley A. Johnson $\mathrm{CD}$ :Systematic review of the side effects associated with tyrosine kinase inhibitors used in the treatment of gastrointestinal stromal tumors on behalf of the EORTC Quality of Life Group. Critical Reviews in Oncology Hematology 91(1):35-46,2014.

6. Holdsworth $\mathrm{CH}$, Badawi RD, Manola JB, Kijewski MF, Israel DA, Demetri GD. Van den Abbeele AD:CT and PET: early prognostic indicators of response to imatinib mesylate in patients with gastrointestinal stromal tumor. Am J Roentgenol 189(6):W324-330,2007.

7. Cassier PA, Blay JY. Imatinib mesylate for the treatment of gastrointestinal stromal tumor. Expert Rev Anticancer Ther. 2010;10(5):623-34.

8. Fletcher CD, Berman JJ, Corless C, Gorstein F, Lasota J, Longley BJ, Miettinen M, O'Leary TJ, Remotti $\mathrm{H}$, Rubin BP, et al: Diagnosis of gastrointestinal stromal tumors: a consensus approach. Hum Pathol 33(5):459-465,2002.

9. Miettinen M, Lasota J. Gastrointestinal stromal tumors: pathology and prognosis at different sites. Semin Diagn Pathol 23(2):70-83,2006.

10. Joensuu H. Risk stratification of patients diagnosed with gastrointestinal stromal tumor. Hum Pathol 39(10):1411-1419,2008.

11. Zhou Y, Hu W, Chen P, Abe M, Shi L, Tan SY, Li Y, Zong L. Ki67 is a biological marker of malignant risk of gastrointestinal stromal tumors. Medicine. 2017;96(34):e7911.

12. Zhi X, Jiang B, Yu J, Røe OD, Qin J, Ni Q, Sun L, Xu M, Zhu J. Ma L:Prognostic role of microscopically positive margins for primary gastrointestinal stromal tumors: a systematic review and meta-analysis. Scientific Reports 6:21541,2016.

13. Mantovani A,Allavena P,Sica A,Balkwill F. Cancer-related inflammation. Nature 454(7203):436$444,2008$.

14. Feng F, Tian Y, Liu S, Zheng G, Liu Z, Xu G, Guo M, Lian X, Fan D, Zhang H. Combination of PLR, MLR, MWR, and Tumor Size Could Significantly Increase the Prognostic Value for Gastrointestinal Stromal Tumors. Medicine. 2016;95(14):e3248. 
15. Atila K, Arslan NC, Derici S, Canda AE, Sagol O, Oztop I. Bora S:Neutrophil-to-lymphocyte ratio: could it be used in the clinic as prognostic marker for gastrointestinal stromal tumor? Hepatogastroenterology 61(134):1649-1653,2014.

16. Racz JM, Cleghorn MC, Jimenez MC, Atenafu EG, Jackson TD, Okrainec A, Venkat Raghavan L. Quereshy FA:Predictive ability of blood neutrophil-to-lymphocyte and platelet-to-lymphocyte ratios in gastrointestinal stromal tumors. Ann Surg Oncol 22(7):2343-2350,2015.

17. Goh BK, Chok AY, Allen JC Jr, Quek R, Teo MC, Chow PK, Chung AY, Ong HS. Wong WK:Blood neutrophil-to-lymphocyte and platelet-to-lymphocyte ratios are independent prognostic factors for surgically resected gastrointestinal stromal tumors. Surgery 159(4):1146-1156,2016.

18. Onodera T, Goseki N, Kosaki G. Prognostic nutritional index in gastrointestinal surgery of malnourished cancer patients. Nihon Geka Gakkai Zasshi 85(9):1001-1005,1984.

19. Migita K, Takayama T, Saeki K, Matsumoto S, Wakatsuki K, Enomoto K, Tanaka T, Ito M, Kurumatani $\mathrm{N}$, Nakajima $Y$. The prognostic nutritional index predicts long-term outcomes of gastric cancer patients independent of tumor stage. Annals of Surgical Oncology 20(8):2647-2654,2013.

20. Hubbard TJE, Lawson-Mclean A, Fearon KC. Nutritional predictors of postoperative outcome in pancreatic cancer. British Journal of Surgery 98(2):268-274,2011.

21. Nozoe T, Kohno M, Iguchi T, Mori E, Maeda T, Matsukuma A, Ezaki T. The prognostic nutritional index can be a prognostic indicator in colorectal carcinoma. Surgery Today 42(6):532-535,2011.

22. Nozoe T, Kimura Y, Ishida M, Saeki H, Korenaga D, Sugimachi K:Correlation of pre-operative nutritional condition with post-operative complications in surgical treatment for oesophageal carcinoma. European Journal of Surgical Oncology 28(4):396-400,2002.

23. Sun J, Mei Y, Zhu Q, Shou C, Tjhoi WEH, Yang W, Yu H, Zhang Q, Liu X, Yu J. Relationship of prognostic nutritional index with prognosis of gastrointestinal stromal tumors. J Cancer. 2019;10(12):2679-86.

24. Joensuu H, Vehtari A, Riihimäki J, Nishida T, Steigen SE, Brabec P, Plank L, Nilsson B, Cirilli C, Braconi C,et al: Risk of recurrence of gastrointestinal stromal tumour after surgery: an analysis of pooled population-based cohorts. Lancet Oncol 13(3):265-274,2012.

25. Gold JS, Gönen M, Gutiérrez A, Broto JM, García-del-Muro X, Smyrk TC, Maki RG, Singer S, Brennan $\mathrm{MF}$, Antonescu CR,et al: Development and validation of a prognostic nomogram for recurrence-free survival after complete surgical resection of localised primary gastrointestinal stromal tumour: a retrospective analysis. Lancet Oncol 10(11):1045-1052,2009.

26. Joensuu H, Rutkowski P, Nishida T, Steigen SE, Brabec P, Plank L, Nilsson B, Braconi C, Bordoni A, Magnusson MK,et al: KIT and PDGFRA Mutations and the Risk of GI Stromal Tumor Recurrence. Journal of Clinical Oncology 33(6):634-642,2015.

27. Ihara K, Yamaguchi S, Shida Y, Fujita J, Matsudera S, Kikuchi M, Muroi H, Nakajima M, Sasaki K, Tsuchioka T, et al: Nutritional status predicts adjuvant chemotherapy outcomes for stage III colorectal cancer. J Anus Rectum Colon 3(2):78-83,2019. 
28. Kang WM, Zhu CZ, Yang XX, Yu JC, Ma ZQ, Ye X, Li K, Liu D. Application of the Onodera prognostic nutrition index and neutrophil-to-lymphocyte ratio in risk evaluation of postoperative complications in Crohn's disease. Sci Rep. 2017;7(1):8481.

\section{Figures}
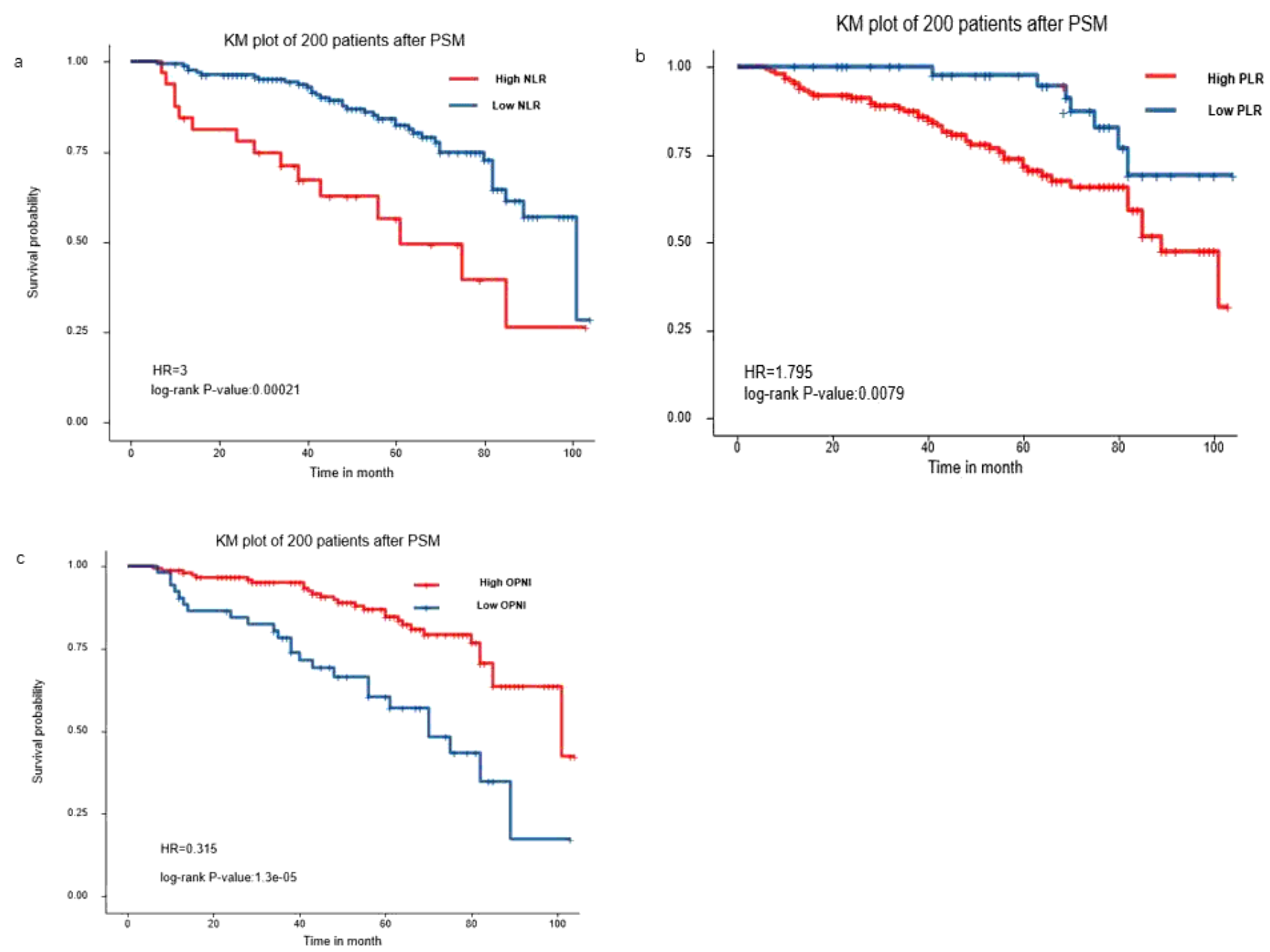

\section{Figure 1}

ROC analysis of NLR (1 a), PLR (1 b) and OPNI (1 c) in TKIs-unused patients. The PLR, NLR, OPNI cut-off value was determined by $\mathrm{R}$ 3.6.3 which was performed based on the recurrence state at 9-year follow-up. And the cut-off point of OPNI is $42.1(P<0.001)$, NLR is $5.1(P<0.001)$ and PLR is $98.6(P=0.008)$. 
a
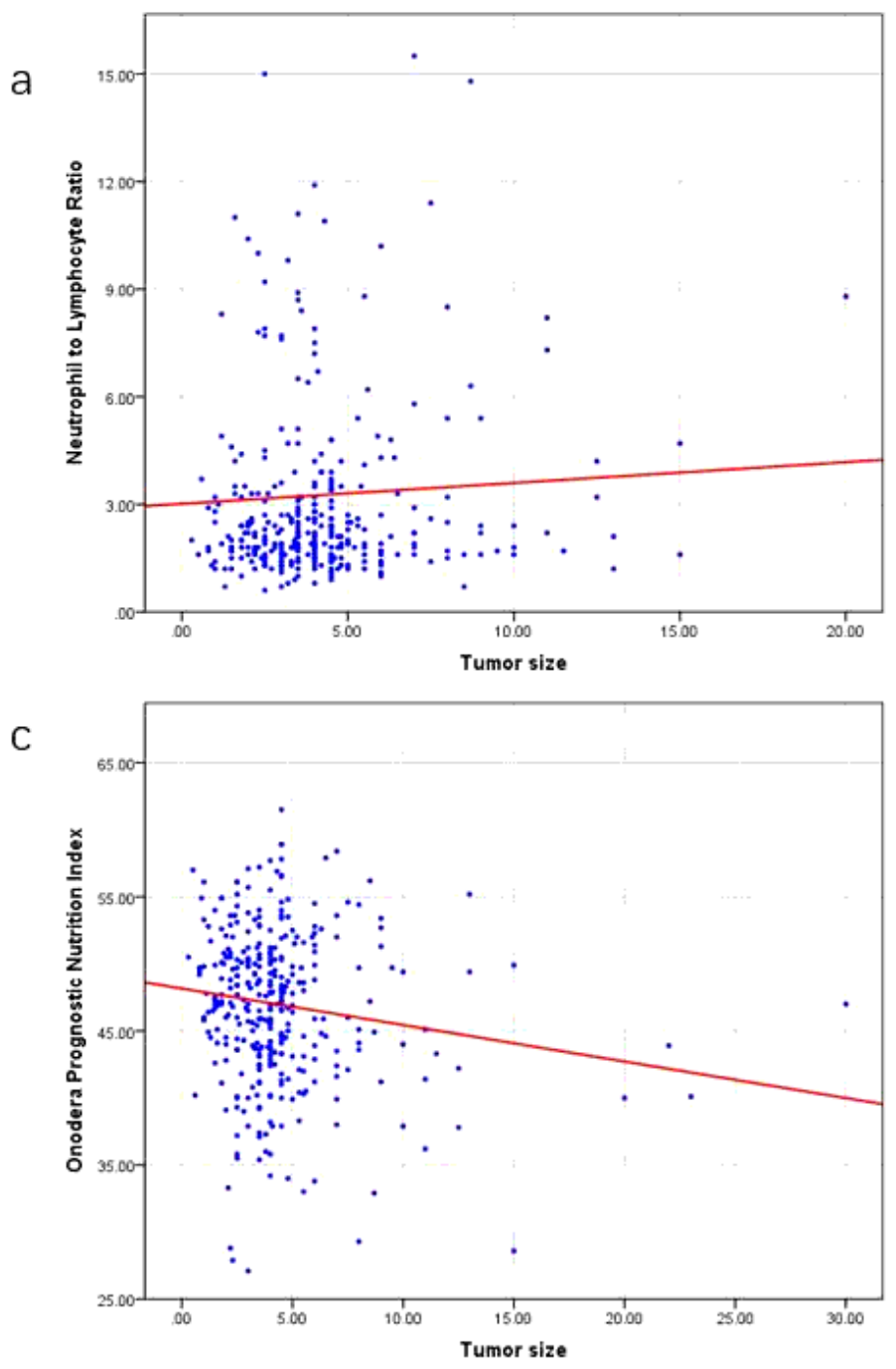
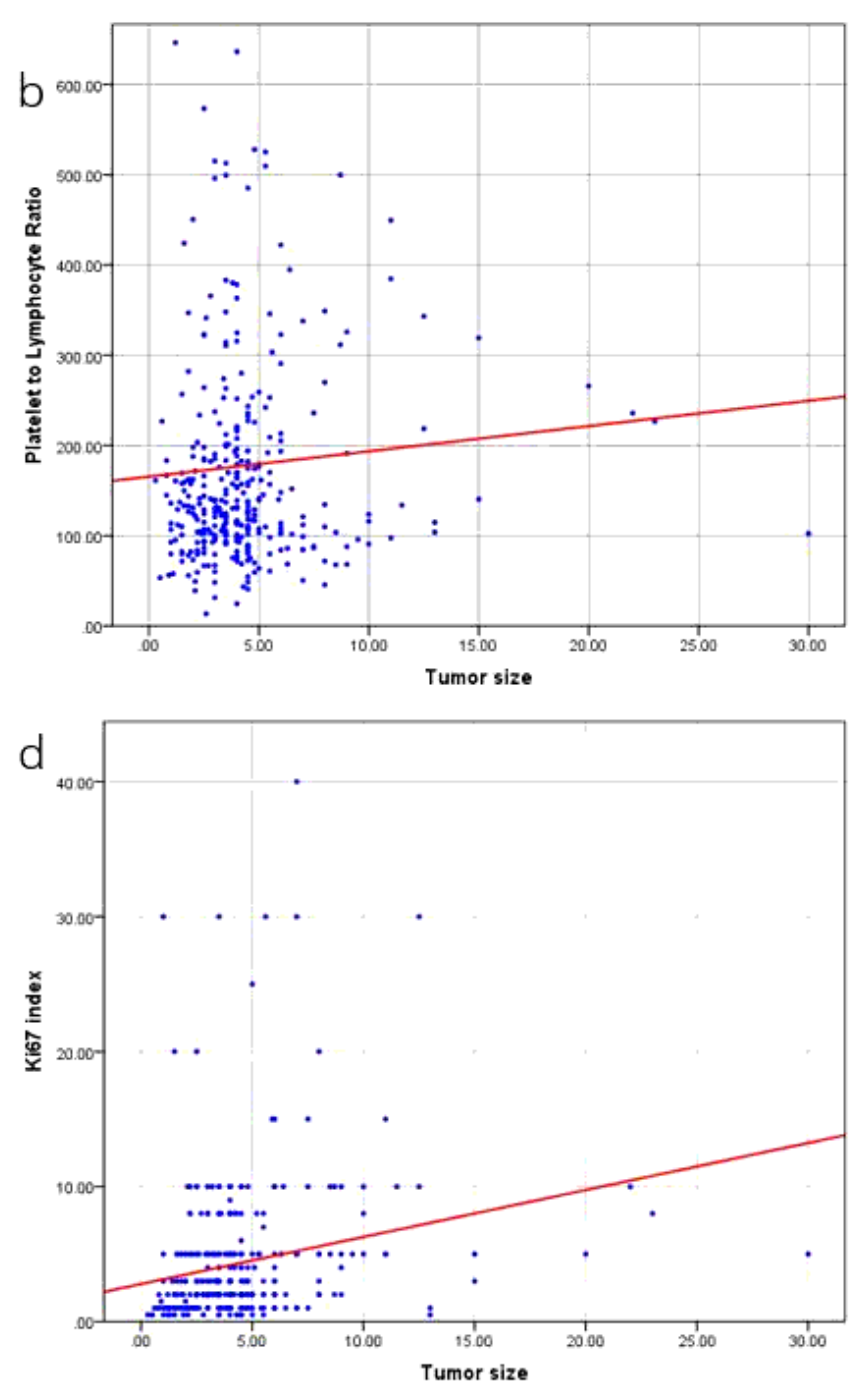

\section{Figure 2}

Correlation between tumor size and NLR (2 a), PLR (2 b), OPNI (2 c) and Ki-67 index (2 d). The correlation of continuous variables was calculated by Pearson correlation coefficient, while discrete variables by Spearman's correlation coefficient. higher OPNI was associated with primary tumor site of stomach $(\mathrm{P}<$ $0.01)$, smaller tumor size $(P<0.017)$, lower mitotic index $(P<0.001)$, lower modified NIH risk classification $(P<0.001)$, less gastrointestinal bleeding rate $(P<0.01)$ and tumor rupture $(P<0.01)$ and much lower tumor relapse rate $(P<0.01)$. 

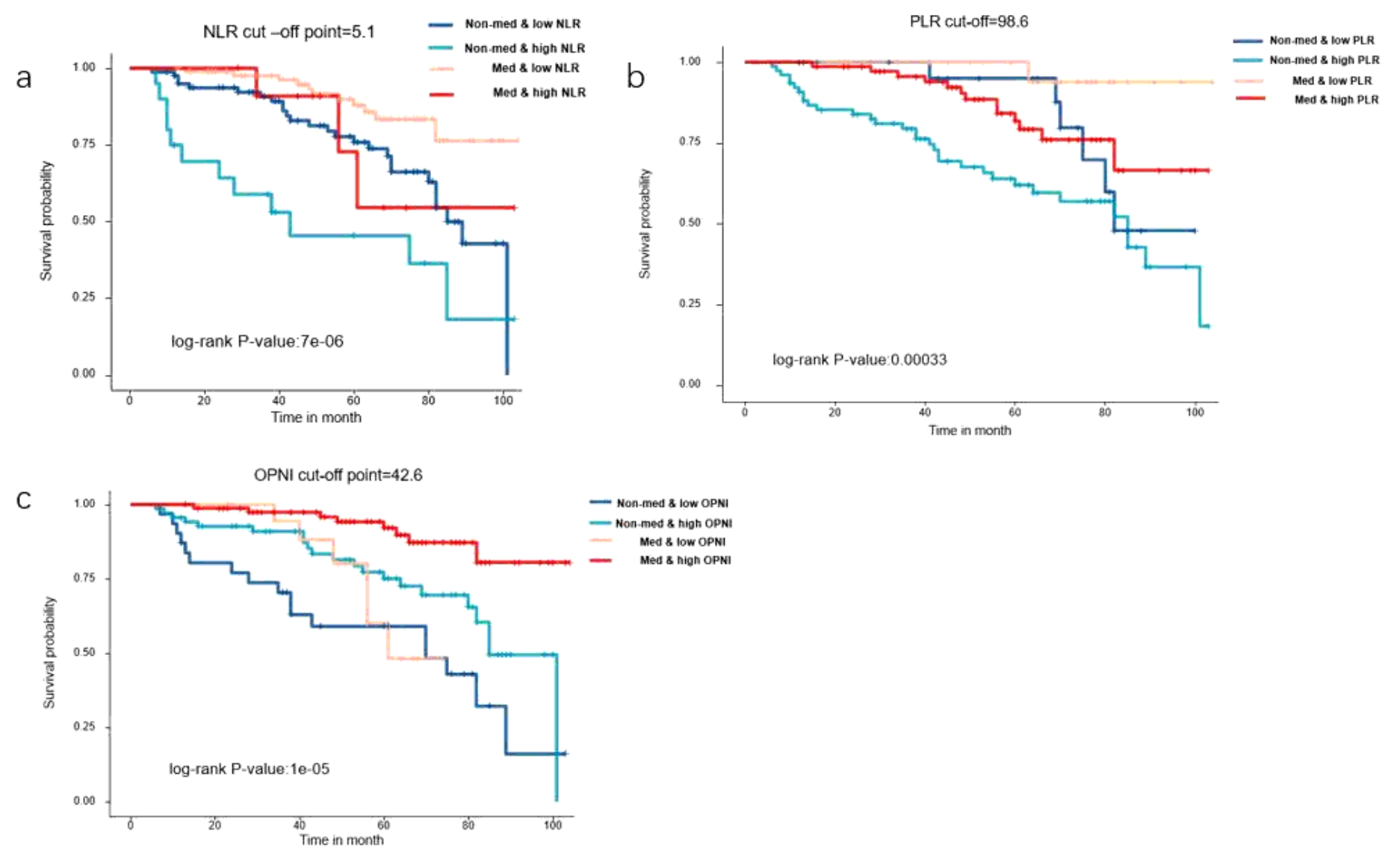

Figure 3

Recurrence-free survival analysis of 200 patients after PSM. Kaplan-Meier curve analysis demonstrated a worse relapse-free survival for patients presenting with ( $3 a$ ) higher NLR, (3 b) higher PLR, and (3 c) lower OPNI. Patients treated with TKIs had better prognosis in our study and low NLR, low PLR and high OPNI also indicated better prognosis. 